\title{
Curvas de referência de pontos brutos no Stanford-Binet Intelligence Scale de crianças e adolescentes
}

\author{
Mária Regina Fumagalli Martdeto- UniversidadeNovedeJulho, SãoPaulo, SãoPaulo, Brasil \\ Terea Hdena Shoen-Fereira - UniversidadeFederal deSãoPaulo, SãoPaulo SãoPaulo, Brasil \\ Brasilia Maria Chian - UniversidadeFedaral deSãoPaulo, SãoPaulo, SãoPaulo Brasil \\ JagyPeissinto- UnivesidadeFedeal deSãoPaulo, SãoPaulo, SãoPaulo, Brasil
}

\begin{abstract}
Resumo
O trabalho teve como objetivo construir curvas de referência de pontos brutos das Áreas e do Total do Stanford-Binet em crianças e adolescentes paulistanos. Foram avaliadas individualmente 257 crianças e adolescentes, com idade média de 5 anos e 10 meses, sendo 130 (50,58\%) do sexo feminino e 127 (49,42\%) do sexo masculino, todas frequentadoras de Escolas Públicas de Educação Infantil e Fundamental, de diferentes regiões da cidade de São Paulo. 0 teste foi aplicado individualmente na própria escola das crianças, sempre a partir do primeiro item, independentemente da idade da criança. Os participantes foram agrupados por idade; calcularam-se medidas descritivas para cada faixa etária desta população. Foram confeccionadas curvas de referência para Áreas e Total do Stanford Binet com os pontos brutos obtidos. Os pontos brutos foram distribuídos de acordo com a curva normal.
\end{abstract}

Palavasdhave Quociente de inteligência; Medidas de inteligência; Inteligência.

\section{Curves reference crude scores in Stanford-Binet Intelligence Scale for children and adolescents}

\begin{abstract}
The objective of this study was to construct curves reference crude scores on the areas and total of the Stanford-Binet test for children in Sao Paulo. 257 children [130 (50.58\%) females and 127 (49.42\%) males] with a mean age of 5 years 10 months were individually evaluated, all of them attending public Nursery and Elementary schools in different regions of the city of São Paulo. The test was applied individually in the children's schools, always based on the first item, regardless of the child's age. The participants were grouped according to age and descriptive measures were performed for each age group in this population. References curves were constructed for the areas and total of the Stanford-Binet test with the crude scores obtained. The crude scores were distributed in accordance with the normal curve.
\end{abstract}

Keywards Intelligence quotient; Intelligence measures; Intelligence.

\section{Curvas de referencia de puntaje bruto en el Stanford-Binet Intelligence Scale de niños y adolescentes}

\begin{abstract}
Resumen
El estudio tuvo como objetivo construir curvas de referencia de puntajes brutos de las Áreas y del Total del StanfordBinet en niños y adolescentes del estado de São Paulo-Brasil. Fueron evaluados individualmente 257 niños y adolescentes, con edad media de 5 años y 10 meses, siendo 130 (50,58\%) del sexo femenino y 127 (49,42\%) del sexo masculino, todos frecuentadores de escuelas públicas de educación infantil y básica, de diferentes regiones de la ciudad de São Paulo. El test fue aplicado individualmente en la propia escuela de los niños, siempre a partir del primer ítem, independientemente de la edad del niño. Los participantes fueron agrupados por edad; se calculó medidas descriptivas para cada rango etario de esta población. Fueron confeccionadas curvas de referencia para Áreas y Total del Stanford Binet con los puntajes brutos obtenidos. Los puntajes brutos fueron distribuidos de acuerdo con la curva normal.

Palabras dave Cociente de inteligencia; Medidas de inteligencia; Inteligencia.
\end{abstract}

Os testes psicológicos são instrumentos técnicos, cuja construção e aplicação necessitam atender a certas condições. Além disso, é necessário considerar características do instrumento no momento da testagem, o objetivo, a relevância de uso de um determinado instrumento para a resolução de um problema, seus aspectos positivos, exigências ou requisitos (Urbina, 2007).

Para Pasquali (2001), quatro parâmetros necessitam ser analisados na aplicação de um teste psicológico: a validade, a precisão ou fidedignidade, a padronização e a normatização. Segundo Anastasi e Urbina (2000), a validade "refere-se àquilo que um teste mede e quão bem ele faz isso", portanto, um teste é válido quando mede o que 0 pesquisador deseja e pensa que está medindo. A precisão ou fidedignidade é conceituada como consistência dos escores obtidos pelas mesmas pessoas quando elas são examinadas com o mesmo teste em diferentes momentos ou por diferentes pessoas no mesmo momento (Pasquali, 2001). Sendo assim, quanto maior a correlação entre os escores dos dois momentos diferentes, maior será a precisão do teste (Anastasi \& Urbina, 2000). A padronização de um teste, que diz respeito à uniformidade de procedimentos utilizados em sua aplicação (Pawlowski, Trentini \& Bandeira, 2007), possui peculiaridades, tais como procedimentos de aplicação, direitos dos testados, controle das variáveis, normas na divulgação dos resultados, as quais garantem a boa qualidade na coleta dos dados, seu uso adequado e eficaz e conclusões coerentes (Noffs, Yazigi, Pascalicchio, Caboclo \& Yacubian, 2006; Noronha, Beraldo \& O liveira, 2003). A normatização permite situar o testando em um padrão ou norma, atribuindo 
sentido aos escores obtidos no teste (Joly, Silva, Nunes \& Souza, 2007; Pasquali, 2001). A padronização implica a uniformidade do processo de avaliação do teste e a normatização, a uniformidade na interpretação dos escores. Os escores brutos devem ser convertidos em medidas relativas para indicar a posição do indivíduo na amostra normativa, avaliando seu desempenho em relação a outras pessoas, e oferecer medidas que permitam comparação direta do desempenho do indivíduo em testes diferentes (Anastasi \& Urbina, 2000; Pasquali, 2001). A normatização é uma das etapas da padronização (Ottati, Noronha \& Salviatti, 2003).

Segundo Vasconcelos (2000), outra maneira de estabelecer normas para um instrumento é por meio de curvas de referência. Um padrão ou curva de referência é a representação sumarizada dos limites de normalidade de um indivíduo (Roberts \& Dallal, 2001; Soares, 2003), ou seja, servem, a um só tempo, para classificar e comparar grupos de referência e para separar indivíduos normais dos que não estão se desenvolvendo dentro do esperado para sua faixa etária (Ferreira, 2000). As curvas de referência, conforme Soares (2003), representam o modelo empírico normal e são construídas com base na observação de medidas dos indivíduos que vivem em condições socioeconômico, culturais e ambientais adequadas ao seu desenvolvimento. Na área de saúde, elas são construídas com 0 objetivo de descrever 0 comportamento da variável resposta de interesse segundo uma variável explicativa em uma população de referência.

As curvas de referência vêm sendo recomendadas desde quando o National Center for Health Statistics (O rganização Mundial de Saúde [O MS], 1995) divulgou para os Estados Unidos, um referencial antropométrico para crianças de 0 a 18 anos de idade de ambos os sexos. A Organização Mundial da Saúde (O MS, 1995) reconheceu esse referencial como adequado para avaliação de diferentes grupos raciais e 0 indicou para uso internacional. No Brasil, o mesmo referencial foi adotado pelo Ministério da Saúde (Ferreira, 2000; Vasconcelos, 2000).

Existem duas formas principais para a construção de curvas de referência: método transversal e método longitudinal. No método transversal, o mais utilizado, por ser mais barato e rápido, as curvas são elaboradas a partir de medidas tomadas uma única vez, de uma amostra de determinada população de um país, região ou cidade, mediante a distribuição dos valores médios e de dispersão. No método longitudinal, que demanda longo tempo de seguimento, os dados são tomados do mesmo grupo de pessoas, desde 0 nascimento até a fase adulta (Pasquali, 2001).
Nas curvas de referëncia, os dados podem ser derivados de diferentes locais de estudo (Gibson, 1990; Woodruff \& Duffield, 2000). A interpretação das medidas exige 0 uso de padrões de referência e de pontos de corte definidos; significando o ponto de corte a linha divisória que distingue os que necessitam e os que não necessitam de intervenção (Waterlow, 1992) e podendo ser determinados estatisticamente (Woodruff \& Duffield, 2000). 0 uso das curvas de referência tem se tornado, embora com limitações, 0 modo mais prático e de menor custo para análise de indivíduos e populações, seja em ações clínicas, seja de triagem ou mesmo em pesquisa em diversos campos profissionais, inclusive na psicologia.

Remetendo-se à psicologia, campo de destaque no presente estudo, para se construir curvas de referência para normatização de instrumentos devem-se respeitar tanto as características demográficas utilizadas na padronização como as próprias características do construto a ser medido (Noronha \& cols., 2003, Pasquali, 2001). Nessa perspectiva, faz-se necessário que testes psicológicos apresentem na população brasileira indicadores de rigor metodológico (padronização, confiabilidade e validação) para seu uso tanto em clínica quanto em pesquisa.

Um teste muito utilizado internacionalmente por seus recursos psicométricos (D eary \& Batty, 2007) é o Stanford-Binet Inteligqne Scale 4th Edition (Thorndike, Hagen \& Satler, 1986). O Stanford-Binet é um teste de nível intelectual que tem como finalidade medir a inteligência geral e as habilidades cognitivas específicas de crianças, adolescentes e adultos (Caruso, 2001; D eary, Whalley, Lemmon \& Crawford, 2000; D ezoete, MacArthur \& Tuck, 2003; Mayes \& Calhoun, 2003). Foi originalmente desenvolvido para ajudar a colocar crianças nas classes educacionais apropriadas, sendo muito utilizado para determinar 0 nível do funcionamento intelectual e assistir no diagnóstico de problemas de aprendizagem, atrasos de desenvolvimento e retardo mental (Barnes \& cols., 2006; Cotton, Voudouris \& Greenwood, 2005; Reschly, 2005), e para o planejamento educacional e avaliação neuropsicológica (Spilkin, Ballantyne \& Trauner, 2009).

A quarta edição do Stanford-Binet avalia a inteligência e a considera como única, geral, composta por habilidades cognitivas específicas. Por ser uma escala composta de vários subtestes que avaliam 0 desempenho cognitivo dos dois anos até a idade adulta, permite caracterizar o perfil das habilidades cognitivas em estudos transversais e longitudinais (D eary \& Batty, 2007; Hockenbury \& Hockenbury, 2003; Hughes \& Ensor, 2009). 
Apesar do Stanford-Binet não apresentar propriedades como a padronização, validação e confiabilidade, para sua utilização, verificadas e divulgadas no Brasil, esse instrumento vem sendo utilizado neste país, submetido à tradução e adaptação transcultural, desde 2000 (Ferreira \& Lamonica, 2011; Guimarães, Souza, Montenegro, Cendes \& Guerreiro, 2003; Herzbert \& Mattar, 2008; Miranda \& cols., 2007; Pereira, Kiyomoto, Cardoso \& Oliveira, 2005; Santos \& Bueno, 2003; Santos, Bueno \& Gathercole, 2006; Silva \& Mulick, 2009). O processo da tradução do Stanford-Binet (Thorndike \& cols., 1986) utilizada neste estudo foi realizado por dois tradutores independentes, qualificados e cientes dos objetivos da tradução. A avaliação da tradução inicial foi realizada por uma retroversão do instrumento (badk trandation) traduzido para o idioma português e pela comparação com a versão original do instrumento. Essa etapa foi realizada por um profissional proficiente na língua inglesa e em linguística. Para venificar a adequação da tradução e do badk trandation, houve uma aplicaçãopiloto realizada por Venske (2001), em 35 (trinta e cinco) crianças de ambos os sexos, na faixa etária de 2 a 10 anos, sorteadas entre aquelas frequentadoras de uma escola de Educação Infantil e Ensino Fundamental, cujo resultado no Teste de Triagem de Denver II havia sido normal. Após os resultados dessa aplicação, dois participantes bilíngues do grupo de pesquisa analisaram 0 material selecionando as palavras e 0 sentido das frases para maior adequação à língua portuguesa. A seguir, houve nova comparação com a retrotradução e a versão final, utilizada neste trabalho. Essa versão foi usada em pesquisa para caracterização do desenvolvimento cognitivo de crianças infectadas pelo HIV (Pedromônico, Venske, Duarte \& Succi, 2000; Venske, 2001) e para avaliar o desempenho das habilidades cognitivas de crianças inseridas em escola de ensino regular (Pedromônico, 2001). Em todos os trabalhos realizados por este grupo de pesquisa a pontuação bruta foi utilizada por representar apenas 0 desempenho correto no teste, além disso, propiciou a observação do aumento de acertos ao longo das idades consideradas, concordando, dessa forma, com os resultados obtidos por Soares (2003). Para dar continuidade à verificação do rigor psicométrico do Stanford Binet, como um instrumento de referência na cidade de São Paulo, o objetivo deste estudo foi construir curvas de referência de pontos brutos das Áreas e do Total do Stanford-Binet Intdligence Scale4th Edition (Thorndike \& cols., 1986) em crianças e adolescentes paulistanos.

\section{Partiapantes}

\section{Método}

Participaram deste trabalho 257 crianças, das quais 130 (50,58\%) do sexo feminino e 127 (49,42\%) do sexo masculino, com idade média de 5 anos e 10 meses (desvio padrão de 1,95). Todos frequentavam escola de educação infantil ou o ensino fundamental em escolas municipais e estaduais das regiões norte, leste e sul da cidade de São Paulo, com tempo médio de escolarização para os meninos de 40,58 meses e para as meninas de 43,98 meses. Como critérios de inclusão neste trabalho, foram considerados a faixa etária de 3 a 14 anos, a matrícula em escola de ensino regular e 0 consentimento dos pais ou responsáveis. Como critério de exclusão, foi considerada a presença de deficiência auditiva ou visual, relatada pelos pais ou professores.

\section{Instrumento}

O Stanford-Binet Inteliggnce Scale 4th Edtion (Thorndike \& cols., 1986) mede a habilidade cognitiva geral e as habilidades cognitivas específicas a partir dos dois anos até os 65 anos de idade (no Brasil, somente foram encontrados estudos com crianças). É composto por 15 subtestes e fornece uma estimativa do nível cognitivo geral e das habilidades cognitivas nas quatro áreas avaliadas: Raciocínio Verbal, com os subtestes Vocabulário, Compreensão, Absurdos e Relações Verbais; Raciocínio Visual-Abstrato, com os subtestes Análise de Padrões (Cubos), Cópia, Matrizes e Dobrar e Cortar; Raciocínio Quantitativo, com os subtestes Quantitativo, Séries Numéricas e Montagem de Equação; e Memória de Curto Prazo, com os subtestes Memória de Contas, Memória de Sentenças, Memória de $\mathrm{O}$ bjetos e Memória para Dígitos.

Todos os subtestes são divididos em níveis a partir dos dois anos de idade. Cada nível de idade é determinado por dois itens de aproximadamente igual dificuldade. Inicia-se a aplicação com o subteste Vocabulário, que utiliza a idade cronológica e, a partir da nota alcançada pela criança, determina a idade mental. A idade mental definirá o ponto de início das outras provas; caso a criança erre um dos itens, a aplicação deverá voltar aos itens dos níveis anteriores até 0 acerto de dois itens consecutivos. A aplicação continua até a criança errar consecutivamente 3 dos 4 itens ou os 4 itens para dois níveis consecutivos.

$\mathrm{Na}$ padronização americana, a pontuação bruta obtida em cada subteste é convertida em escores padronizados por idade, por meio de tabelas correspondentes. Quando a média é igual a 100 e 0 desvio padrão igual a 16, encontra-se 0 escore composto (SAS) considerado normal. Esse escore equivale ao quociente de inteligência (QI) ou capacidade de raciocínio geral. 


\section{Hrocementodecolea decados}

Após a aprovação pelo Comitê de Ética em Pesquisa da Universidade Federal de São Paulo Unifesp, com a devida autorização dos serviços envolvidos, bem como com a assinatura do Termo de Consentimento Livre e Esclarecido pelo responsável (CEP n- 0334/ 06), realizou-se nas escolas um encontro coletivo com pais e professores, a fim de pedir-lhes autorização para avaliar seus filhos. Após 0 consentimento das instituições e dos responsáveis, iniciou-se a avaliação do nível intelectual das crianças e adolescentes no próprio estabelecimento educacional e no horário escolar. Todos foram avaliados individualmente pelo teste de nível intelectual. Com 0 intuito de obter 0 perfil Total e por Áreas do instrumento, a aplicação em todas as crianças iniciou-se com o subteste Vocabulário, no item 1. Os outros subtestes foram aplicados partindo-se também do item 1. Neste estudo, aplicaram-se 10 (dez) subtestes do Stanford-Binet que correspondem à faixa de idade da população estudada.

\section{Análisededados}

Após a avaliação e levantamento dos pontos brutos dos protocolos, foi montado um único banco de dados, contendo os pontos brutos dos subtestes do Stanford-Binet e suas respectivas áreas por sexo e por idade, considerando todas as crianças. Foram feitas medidas descritivas para identificar os escores médios para cada idade dessa população.
As crianças e adolescentes foram agrupados por idade e por sexo, e foram confeccionadas curvas de referência (Boyd \& Harris, 1995) por Áreas e Total do Stanford-Binet com todos os pontos brutos obtidos. Os pontos brutos foram considerados variáveisresposta e a idade e sexo variáveis explicativas. $\mathrm{Na}$ construção das curvas, foi adotado 0 método paramétrico, que supõe que as distribuições sejam normais, com a mesma variância, e foram construídas curvas de referência de 5\%,50\% e $95 \%$ para a população de referência.

\section{Resultados}

A Tabela 1 apresenta a distribuição das crianças e adolescentes por regiões, segundo o sexo, a idade, escolaridade materna e a renda familiar.

Os resultados da Tabela 1 indicam que houve maior avaliação de crianças de 3 a 8 anos na Zona Leste e de 9 a 14 anos na Zona Norte. Com relação ao sexo, as crianças se distribuíram de modo equivalente nas três regiões da cidade de São Paulo inseridas neste estudo. A escolaridade materna até 8 anos foi maior na região Norte da cidade, com cerca de $50 \%$ das mães apresentando essa escolaridade. Com relação à renda familiar, nas três regiões, $78 \%$ da população apresentou renda entre 0 e dois (2) salários-mínimos e cerca de 15\% entre dois (2) e cinco (\%) salários-mínimos. A renda tendeu de baixa à precária na população deste estudo.

Tabela 1. Distribuição, em porcentagem, do sexo, idade, escolaridade materna e renda familiar por região da cidade de São Paulo

\begin{tabular}{lcccc}
\hline & & Zona Leste (\%) & Zona Sul (\%) & Zona Norte (\%) \\
\hline Sexo & Masculino & 43,57 & 61,76 & 46,94 \\
& Femininos & 56,43 & 38,24 & 53,06 \\
Idade & 3 a 5 anos & 72 & 54 & 0 \\
& 6 a 8 anos & 78 & 14 & 21 \\
& 9 a 11 anos & 0 & 0 & 18 \\
& 12 a 14 anos & 0 & 0 & 9 \\
\hline Escolaridade materna & (até 8 anos) & 24,90 & 18,50 & 57,70 \\
Renda familiar & (até 1,5 salário-minimo) & 77,26 & 79,10 & 76,09 \\
\hline
\end{tabular}

Considerando as curvas de referência construídas, em todos os diagramas de dispersão, os pontos de corte nos percentis 5 e 95 são apresentados por idade, em cada área do Stanford-Binet.

A Figura 1 apresenta o diagrama de dispersão do Raciocínio Verbal em pontos brutos e a variável idade na amostra de crianças e adolescentes normais sobreposta às curvas de referência.

O bservamos que, por exemplo, para a idade de 4 anos esperamos que $5 \%$ da população tenha escore menor ou igual a 9 e 5\% da população escore igual ou maior que 31 no Raciocínio Verbal. 


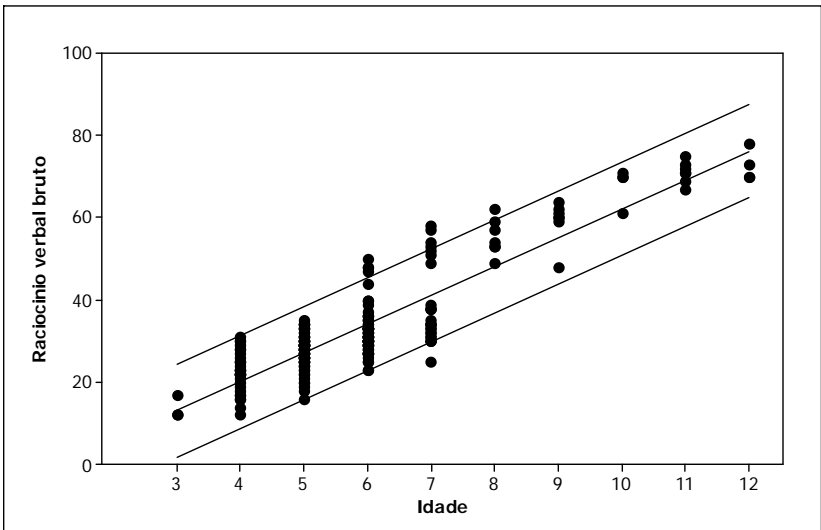

Figura 1. Diagrama de dispersão do Raciocínio Verbal em pontos brutos por idade e curvas de referência de $5 \%, 50 \%$ e $95 \%$ na população

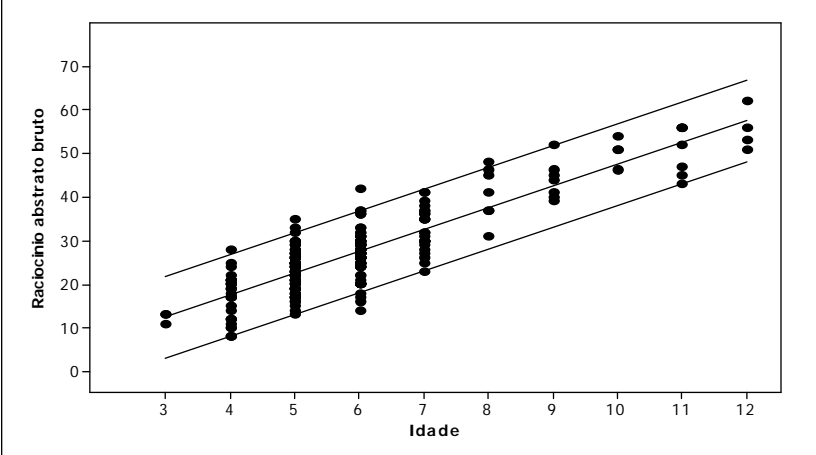

Figura 2. Diagrama de dispersão do Raciocínio VisualAbstrato em pontos brutos por idade e curvas de referência de 5\%, 50\% e 95\% na população

A Figura 2 apresenta o diagrama de dispersão do Raciocínio Visual-Abstrato em pontos brutos e a variável idade na amostra de crianças e adolescentes normais sobreposta às curvas de referência. Observamos que, por exemplo, para a idade de 4 anos esperamos que $5 \%$ da população tenha escore menor ou igual a 8 e 95\% da população escore igual ou maior que 27 no Raciocínio Visual-Abstrato.

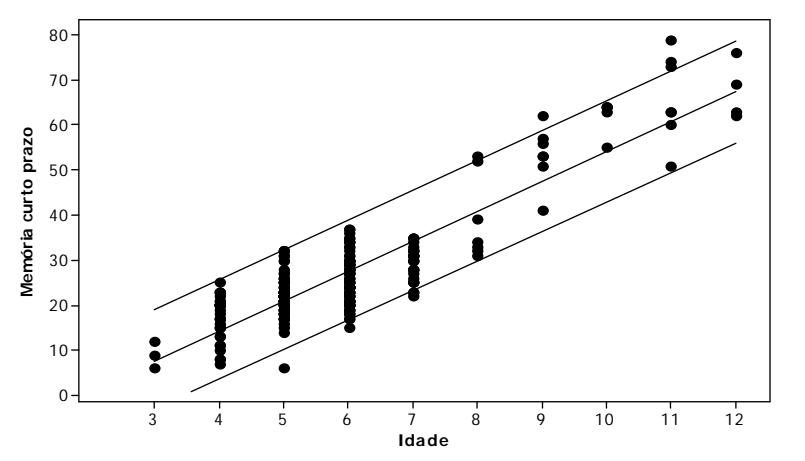

Figura 3. Diagrama de dispersão da Memória por idade e curvas de referência de $5 \%$, 50\% e 95\% na população
A Figura 3 apresenta 0 diagrama de dispersão da Área Memória em pontos brutos e a variável idade na amostra de crianças e adolescentes normais sobreposta às curvas de referência. O bservamos que, por exemplo, para a idade de 4 anos esperamos que $5 \%$ da população tenha escore menor ou igual a 3 e $95 \%$ da população escore igual ou maior que 26 para a Memória.

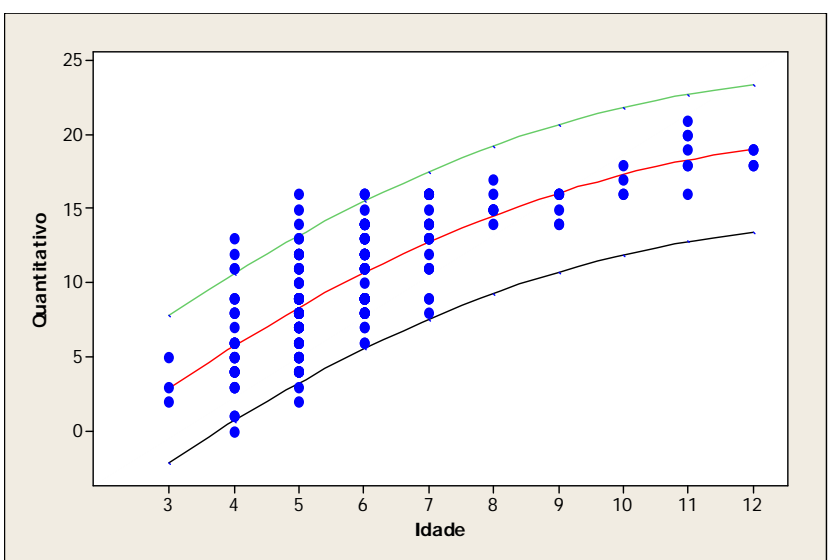

Figura 4. Diagrama de dispersão do Raciocínio Quantitativo por idade e curvas de referência de $5 \%$, $50 \%$ e $95 \%$ na população

A Figura 4 apresenta o diagrama de dispersão da área Raciocínio Quantitativo em pontos brutos e a variável idade na amostra de crianças e adolescentes normais sobreposta às curvas de referência. Observamos que, por exemplo, para a idade de 4 anos esperamos que $5 \%$ da população tenha escore menor ou igual a 1 e 95\% da população escore igual ou maior que 11 para o Raciocínio Quantitativo.

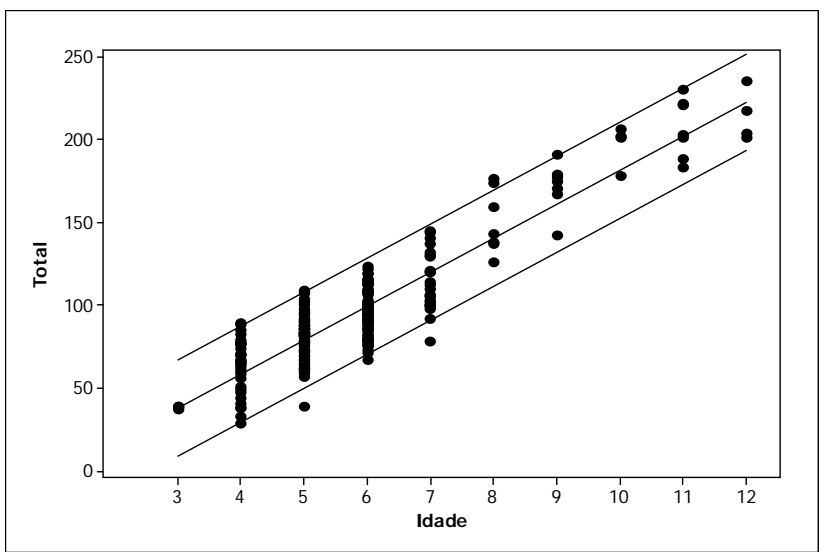

Figura 5. Diagrama de dispersão do Total em pontos brutos por idade e curvas de referência de 5\%, $50 \%$ e $95 \%$ na população

A Figura 5 apresenta 0 diagrama de dispersão do Total em pontos brutos e a variável idade na amostra de crianças e adolescentes normais sobreposta às curvas de referência Observamos que, por exemplo, 
para a idade de 4 anos esperamos que $5 \%$ da população tenha escore menor ou igual a 29 e 95\% da população escore igual ou maior que 88 para o Total.
A Tabela 2 apresenta os pontos no percentil $5 \mathrm{e}$ 95, em cada faixa etária.

Tabela 2. Pontos de corte nos percentis 5 e 95, por idade, em cada área do Stanford-Binet

\begin{tabular}{cccccc}
\hline \multirow{2}{*}{ Idade } & Raciocínio Verbal & $\begin{array}{c}\text { Raciocínio Visual } \\
\text { Abstrato }\end{array}$ & $\begin{array}{c}\text { Raciocínio } \\
\text { Quantitativo }\end{array}$ & Memória & Total \\
\cline { 2 - 6 } & $5 \% / 95 \%$ & $5 \% / 95 \%$ & $5 \% / 95 \%$ & $5 \% / 95 \%$ & $5 \% / 95 \%$ \\
\hline 3 anos & $2 / 24$ & $3 / 22$ & $0 / 8$ & $0 / 19$ & $9 / 67$ \\
4 anos & $9 / 31$ & $8 / 27$ & $1 / 11$ & $3 / 26$ & $29 / 88$ \\
5 anos & $16 / 38$ & $13 / 32$ & $3 / 13$ & $9 / 32$ & $50 / 108$ \\
6 anos & $23 / 45$ & $18 / 37$ & $6 / 16$ & $16 / 39$ & $70 / 129$ \\
7 anos & $30 / 52$ & $23 / 42$ & $8 / 18$ & $23 / 45$ & $91 / 149$ \\
8 anos & $37 / 59$ & $28 / 47$ & $9 / 19$ & $29 / 52$ & $111 / 170$ \\
9 anos & $44 / 66$ & $33 / 52$ & $11 / 21$ & $36 / 59$ & $132 / 190$ \\
10 anos & $51 / 73$ & $38 / 55$ & $12 / 22$ & $43 / 65$ & $152 / 211$ \\
11 anos & $58 / 80$ & $43 / 62$ & $13 / 23$ & $49 / 72$ & $173 / 231$ \\
12 anos & $65 / 87$ & $48 / 67$ & $13 / 23$ & $56 / 79$ & $193 / 252$ \\
\hline
\end{tabular}

\section{Discussão}

Com a finalidade de descrever o desempenho cognitivo de crianças e adolescentes, inseridos em escolas de ensino regular, no teste Stanford-Binet Intellignne Scale 4th Edition (Thornike \& cols., 1986), foram confeccionadas curvas de referência (Boyd \& Harris, 1995) por Áreas e Total do teste, com todos os pontos brutos obtidos. Procurou-se, neste estudo, obter curvas de referência do desempenho cognitivo aplicando o instrumento em crianças com as mesmas condições socioeconômico-culturais em diferentes regiões da cidade de São Paulo. É preciso ressaltar, no entanto, que uma das limitações deste estudo está no fato de que as crianças e adolescentes avaliados representam somente níveis socioeconômicos médiobaixo e baixo, sendo este um fator importante a ser considerado em novas pesquisas, ou seja, faz-se necessário aplicar o Teste de Nível Intelectual Stanford-Binet em crianças e adolescentes frequentadores de escolas particulares.

Neste estudo, os escores brutos não foram transformados em medidas relativas, mas classificaram as crianças e adolescentes dentro da faixa de normalidade de $5 \%$ e $95 \%$. Os dados antropométricos, sexo, idade e pontos brutos do Stanford Binet foram derivados da aplicação do instrumento em escolas de ensino infantil e fundamental em três regiões da cidade de São Paulo, conforme sugere a literatura (Godoy \& cols., 2005; Schoen-Ferreira, Meneguelli \& AznarFarias, 2005). Encontraram-se estatisticamente pontos de corte definidos que possibilitam a classificação e interpretação de novas pontuações obtidas, bem como situar 0 ponto bruto obtido por crianças e adolescentes, dentro dessas curvas, indicando assim 0 seu desempenho intelectual de acordo com recomendações internacionais.

Dessa forma, a construção das curvas para 0 Stanford-Binet Intdligenescale4th Edition(Thorndike \& cols., 1986) apresenta uma etapa da normatização do teste para a população paulistana, ou seja, a pontuação bruta obtida por uma criança diante da aplicação do instrumento possibilita comparar o seu desempenho com o de outras crianças da mesma faixa etária. Diante disso, percebe-se a necessidade de que instrumentos produzidos em países diferentes sofram estudos de padronização e normatização para cada lugar onde serão utilizados. Essa preocupação deve existir, já que, ao se interpretarem os resultados do teste, não se usam as mesmas normas que foram utilizadas com populações e culturas diferentes. Cabe aos profissionais, aos pesquisadores, às universidades e às editoras esse cuidado com 0 desenvolvimento científico. Observou-se, também, que a mensuração da inteligência realizada por meio do desempenho no teste Stanford-Binet (Thorndike \& cols., 1986) esteve relacionada à idade da criança. Sendo assim, o estabelecimento de normas para instrumentos destinados à avaliação de nível intelectual de acordo com contextos culturais diferentes faz-se relevante, na medida em que previne ao máximo os equívocos que podem ocorrer ao se usar um material padronizado para línguas e culturas distintas.

\section{Referências}

Anastasi, A., \& Urbina, S. (2000). Tetaggm picdógia Porto Alegre: Artes Médicas.

Barnes, M. A., Wilkinson, M., Khemani, E., Boudesquie, A., Dennis, M., \& Fletcher, J. M. 
(2006). Arithmetic processing in children with spina bifida. Jaumal of Leming Disabilities, 39(2), 174-187.

Boyd, J. C., \& Harris, E. K. (O rg.). (1995). Statistical bases of reference values in laboratary meeliane Nova Iorque: Marcel D ekker.

Caruso, J. C. (2001). Reliable component analysis of the Stanford-Binet: Fourth edition for 2-to 6-yearolds. Psychdogical Assessment, 13, 261-266.

Cotton, S. M., Voudouris, N. J., \& Greenwood, K. M. (2005). Association between intellectual functioning and age in children and young adults with duchenne muscular dystrophy: further results from a meta-analysis. Dexdopmental Meeliane \& ChildNeurdogy, 47(4), 257-265.

Deary, I. J., \& Batty, G. D. (2007). Cognitive epidemiology. Jaumal of Epidemidogy and Community Health, 61(5), 378-384.

D eary, I. J., Whalley, L. J., Lemmon, H., \& Crawford, J. R. (2000). The stability of individual differences in mental ability from childhood to old age: followup of the 1932 scottish mental survey. Intdligence, $28,49-55$

Dezoete, J. A., MacArthur, B. A., \& Tuck, B. (2003) Prediction of Bayley and Stanford-Binet Scores with a group of very low birthweight children. Child: Care, HealthandDeddqumet, 29, 367-372.

Ferreira, A. T., \& Lamonica, D., A. C. (2011). Comparação do léxico de crianças com Síndrome de D own e com desenvolvimento típico de mesma idade mental. Rev. CEFAC [online]. http:/ / www.scielo.br/ scielo.php?script=sci_arttex t\&pid=S1516 $18462011005000041 \& \operatorname{lng}=e n \& n r m=i s o$.

Ferreira, H. S. (2000). Avaliação nutricional de crianças pelo método antropométrico. Em H. S. Ferreira (O rg.), Desnutriçãa magritude significado social e possibilidade de prearção (pp. 33-89). Maceió: EDUFAL.

Gibson, R. S. (1990). Anthropometric reference data. Em R. S. Gibson (O rg.), Prinaples of nutritional assessment (pp.209-46). Nova Iorque: Oxford University Press.

Godoy, Y., Wehba, J., Pasquali, L., \& Aznar-Farias, M. (2005). Validação do Questionário de Identidade para pré-adolescentes-QI-PREAD . Em Anais do II Congresso Brasileiro de Avaliação Psicdóġa - Desafios para a Fomação Prática e Pesquisa Gramado-RS: Instituto Brasileiro de Avaliação Psicológica
Guimarães, C. A., Souza, E. A. P., Montenegro, M. A., Cendes, F., \& Guerreiro, M. (2003). Avaliação neuropsicológica e de qualidade de vida. Arquivos deNeurqpsiquiatria, 61(3-B), 786-792.

Herzbert, E., \& Mattar, A. (2008). Instrumentos clínicos utilizados no Departamento de Psicologia Clínica da USP: 10 anos depois. BdeimdePsicdoga, 58, 39-54.

Hockenbury, D. H., \& Hockenbury, S. E. (Orgs.). (2003). Desconindba psicdoga Barueri: Manole.

Hughes, C. H., \& Ensor, R. A. (2009). How do families help or hinder the emergence of early executive function? New Diretions for Child and Addescent Deudoment, 123, 35-50.

Joly, M. C. R. A., Silva, M. C. R., Nunes, M. F. O., \& Souza, M. S. (2007). Análise da produção científica em painéis dos congressos brasileiros de avaliação psicológica. AvaliaçãoPsiđdógica, 6, 239-252.

Mayes, S. D., \& Calhoun, S. L. (2003). Analysis of WISC-III, Stanford-Binet: IV, and Academic Achievement Test Scores in children with autism. Joumal of Autism and Deudqumental Disordes, 33, 329-341.

Miranda, M. C., Nóbrega, F. J., Sato, K., Pompéia, S., Sinnes, E. G., \& Bueno, O. F. A. (2007). Neuropsychology and malnutrition: a study with 7 a 10 years-old children in a poor community. Revista Brasileira de Saúde Matemo Infantil, 7(1), 4554.

Noffs, M. H. S., Yazigi, L., Pascalicchio, T. F., Caboclo, L. O. S. F., \& Yacubian, E. M. T. (2006). D esempenho cognitivo de pacientes com epilepsia do lobo temporal e epilepsia mioclônica juvenil: avaliação por meio da escala WAIS III. Jaumal EpilepsyClinical Neurophysidogy, 12(1), 7-12.

Noronha, A. P. P., Beraldo, F. N. M., \& Oliveira, K. L. (2003). Instrumentos psicológicos mais conhecidos e utilizados por estudantes e profissionais de psicologia. Psiđoga Escla Eduraçãa 7(1), 47-56.

Organização Mundial de Saúde. (1995). Physical status the use and interpreation of anthropomtry. Genebra: OMS.

Ottati, F., Noronha, A. P. P., \& Salviatti, M. (2003). Testes psicológicos: qualidade de instrumentos de interesse profissional. Inteação em Psicdoga, 7(1), 65-71. 
Pasquali, L. (2001). Ténicas de exame picdóg்a T.E.P. manual. Fundamentos das ténicas psicoógicas São Paulo: Casa do Psicólogo.

Pawlowski, J., Trentini, C. M., \& Bandeira, D. R. (2007). Discutindo procedimentos psicométricos a partir da análise de um instrumento de avaliação neuropsicológica breve. PsicoUSF, 12(2), 211-219.

Pedromônico, M. (2001). Avaliando 0 desenvolvimento. Arquivos de Neuropsiquiatria, 59, 16-17.

Pedromônico, M. R. M., Venske, S., Duarte, C. S., \& Succi, R. C. M. (2000). Problemas de comportamento em filhos de mães portadoras de HIV. Fdha Médica, 119(2), 29-35.

Pereira, C. C. S., Kiyomoto, B. H., Cardoso, R., \& Oliveira, A. S. B. (2005). Distrofia muscular de Duchenne: imunoexpressão da a-distroglicana em musculatura esquelética e performance cognitiva. Arquivos deNeuro-Psiquiatria, 63, 984-989.

Reschly, D. J. (2005). LD identification: primary intervention, secondary intervention, then what? Jaumal of LeamingDisabilities, 38(6), 510-515.

Roberts, S. B., \& Dallal, G. E. (2001). The new childhood growth charts. Nutrition Reviens, 59(2), 31-35.

Santos, F. H., Bueno, O. F. A., \& Gathercole, S. E. (2006). Errors in nonword repetition: bridgingshort and long-term memory. Brazilian Jamal Meelical BidogyResearch, 39(3), 371-385.

Santos, F. H., \& Bueno, O. F. A. (2003). Validation of the brazilian children's Test of Pseudoword Repetition in portuguese speakers aged 4 to 10 years. Brazilian Jaumal of Medial and Bidogial Rescarch, 36, 1533-1547.

Schoen-Ferreira, T. H., Meneguelli, N., \& Aznar-Farias, M. (2005). Validação semântica do Extended Objective Measure of Ego Identity Status-2EOMEIS-2. Em Anais do Congesso Brasilero de AvaliaçãoPsicdógia, Gramado-RS, Brasil.
Silva, M., \& Mulick, J. J. (2009). Diagnosticando o transtorno autista: aspectos fundamentais e considerações práticas. Psicdoga: Ciênia ePrơissãa 29, 116-131.

Soares, N. T. (2003). A new growth anthropometric reference: meanings and implications. Rexista de Nutriçãa 16(1), 93-104.

Spilkin, A. M., Ballantyne, A. O., \& Trauner, D. A. (2009). Visual and verbal learning in a genetic metabolic disorder. Neuropsydhogia, 47(8-9), 18831892.

Thorndike, R. L., Hagen, E. P., \& Satler, J. M. (1986).

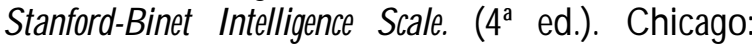
Riverside.

Urbina, S. (2007). Introdução aos testes psicológicos e seus usos. Em S. Urbina (Ed.), Fundamentos da testagmmodkma (pp. 11-41). Porto Alegre: Artmed.

Vasconcelos, F. A. G. (2000). Indicadores antropométricos III. Em F. A. G. Vasconcelos (O rg.), Avaliaçãontricional decdetividades ( $2^{\mathrm{a}}$ ed., pp. 67-81). Florianópolis: DAUFSC.

Venske, S. (2001). Caradteização do desenduimento cognitivo de ciancas infetadas pdo HIV (Dissertação de Mestrado). Universidade Federal de São Paulo, São Paulo, Brasil.

Waterlow, J. C. (1992). Nutrition and growth. Em J. C. Waterlow (Ed.), Protein enegy malnutrition (pp. 187211). Londres: Edward Amold.

Woodruff, B. A., \& Duffield, A. (2000). Assessment of nutritional status in emegrng-affeted pquilations Genebra: United Nations/ Sub-Committee on Nutrition. 
Sobre as autoras:

Márcia Regina Fumagalli Marteleto é psicóloga, mestre em Psicologia Aplicada em Pediatria (Universidade Federal de São Paulo) e doutora em Distúrbios da Comunicação Humana (Universidade Federal de São Paulo). A tualmente é coordenadora e professora do Curso de Psicologia da Universidade Nove de Julho, atuando na área de Psicologia (Psicologia do D esenvolvimento Humano, Psicopatologias do D esenvolvimento e Avaliação Psicológica).

Teresa Helena Schoen é psicóloga, mestre em Ciências Aplicadas à Pediatria (Universidade Federal de São Paulo), doutora em Ciências (Universidade Federal de São Paulo), atuando na área de Psicologia Clínica e do D esenvolvimento, com ênfase na adolescência e construção de identidade.

Brasilia Maria Chiari é fonoaudióloga, professora titular, livre-docente do Departamento de Fonoaudiologia da Universidade Federal de São Paulo, atuando com avaliação, diagnóstico e reabilitação da linguagem nos déficits da audição. É pesquisadora 1D do CNPQ.

Jacy Perissinoto é fonoaudióloga, mestre em Lingüística Aplicada e Estudos da Linguagem (Pontifícia Universidade Católica de São Paulo), doutora em Distúrbios da Comunicação Humana (Universidade Federal de São Paulo) e Pós-D outorado pela Université Paris V - Sorbone. Atualmente é professora na Universidade Federal de São Paulo, atuando na área de Fonoaudiologia (linguagem infantil e do adolescente, autismo, desenvolvimento e avaliação fonoaudiológica da linguagem).

Contato com as autoras:

Rua D r. Miranda de Azevedo, 752, apto 204 - CEP 05027-000 - São Paulo-SP.

E-mail: marcia.marteleto@terra.com.br 